\title{
ERCP improves mortality in acute biliary pancreatitis without cholangitis
}

\section{두)(웅}

\section{Authors}

Aleksey A. Novikov ${ }^{1}$, Jennifer H. Fieber ${ }^{2}$, Monica Saumoy ${ }^{3}$, Russell Rosenblatt ${ }^{4}$, Shirley A. Cohen Mekelburg ${ }^{5}$, Shawn L. Shah ${ }^{4}$, Carl V. Crawford ${ }^{4}$

\section{Institutions}

1 Department of Gastroenterology, Doylestown Hospital, Doylestown, Pennsylvania, United States

2 Department of Surgery, Hospital of the University of Pennsylvania,

Philadelphia, Pennsylvania, United States

3 Division of Gastroenterology and Hepatology, Hospital of the University of Pennsylvania, Philadelphia, Pennsylvania, United States

4 Division of Gastroenterology and Hepatology, Weill Cornell Medical College, New York, New York, United States

5 Gastroenterology and Hepatology, University of Michigan, Ann Arbor, Michigan, United States

submitted 31.1.2020

accepted after revision 23.9.2020

Bibliography

Endosc Int Open 2021; 09: E927-E933

DOI 10.1055/a-1320-0041

ISSN 2364-3722

(C) 2021. The Author(s).

This is an open access article published by Thieme under the terms of the Creative Commons Attribution-NonDerivative-NonCommercial License, permitting copying and reproduction so long as the original work is given appropriate credit. Contents may not be used for commercial purposes, or adapted, remixed, transformed or built upon. (https://creativecommons.org/licenses/by-nc-nd/4.0/)

Georg Thieme Verlag KG, Rüdigerstraße 14,

70469 Stuttgart, Germany

Corresponding author

Dr. Aleksey Novikov, Department of Gastroenterology,

Doylestown Hospital, Doylestown, Pennsylvania,

United States

anovikov@dh.org

Supplementary material is available under

https://doi.org/10.1055/a-1320-0041

\section{ABSTRACT}

Background and study aims Acute pancreatitis (AP) is an increasingly common indication for hospitalization in the United States. The necessity for endoscopic retrograde cholangiopancreatography (ERCP) and the timing of ERCP in acute gallstone-related pancreatitis without cholangitis (AGPNC) is controversial. The aim of this study was to evaluate the association of ERCP and its performance during admission with mortality and length of stay (LOS) in patients with AGPNC.

Patients and methods We queried the Nationwide Inpatient Sample (NIS) from 2004 to 2014 to identify all patients with admissions for gallstone AP. We excluded patients with chronic pancreatitis or concurrent cholangitis, and those who were transferred from elsewhere for treatment. Our primary outcome measure was inpatient mortality. Our secondary outcome measure was hospital length of stay (LOS).

Results We identified 491,011 records eligible for analysis. Of the patients, $30.6 \%(150,101)$ had AGPNC. There were 1.34 deaths per 100 admissions in patients with AGPNC. The average LOS was $5.88( \pm 6.38)$ days with a median stay of 4 days (range, 3-7). When adjusted for age, Elixhauser Comorbidity Index, and severe pancreatitis, patients with ERCP during admission were $43 \%$ less likely to die. ERCP performed between Days 3 and 9 of hospitalization resulted in a significant mortality benefit. Among those who had ERCP, a shorter wait time for ERCP was associated with a shorter LOS after adjustment for demographics and severity of illness.

Conclusion ERCP performed during inpatient admission for AGPNC was associated with decreased mortality. These data support early ERCP in patients with acute gallstone pancreatitis without cholangitis. 


\section{Introduction}

Acute pancreatitis (AP) is the third most common gastroenterology-related indication for hospitalization in the United States, with approximate 270,000 hospital admissions per year and increasing since 2000 [1,2]. Biliary disease remains the most common etiology for AP in Western countries. [1] Despite the increasing recognition of acute pancreatitis and current guidelines for management, severe necrotizing pancreatitis continues to have mortality up to $30 \%$ [2].

Early surgical data and animal models have shown evidence of improved outcomes after biliary decompression in patients with acute gallstone-related pancreatitis without cholangitis (AGPNC) [3-5]. Early studies by Neoptolemos, Fan, and Nowak showed trends toward decreased mortality in AGPNC treated with endoscopic retrograde cholangiopancreatography (ERCP) and sphincterotomy; however, only the study from Nowak's group showed a statistically significant difference [6-8]. A first meta-analysis showed a benefit for early ERCP in gallstone pancreatitis based on four randomized controlled trials (RCTs) available at the time [9]. Thirteen years later, however, a Cochrane group meta-analysis, based on the pooled data from seven RCTs, did not find a significant benefit for ERCP during hospitalization or at 24 and 72 hours after admission [10]. The definitions of gallstone pancreatitis, pancreatitis severity, degree of biliary obstruction, timing of endoscopic intervention, and clinical improvement were markedly different across all studies, weakening overall conclusions from the Cochrane review. Because of study heterogeneity, there is no consensus in the American Gastroenterology Association (AGA), American College of Gastroenterology (ACG), or International Association of Pancreatology (IAP)/American Pancreatic Association (APA) guidelines on the benefit or timing of ERCP for AGPNC [1113]. We aimed to establish whether there is an association with mortality or length of stay (LOS) in patients with AGPNC that underwent ERCP compared to medical management using a large retrospective database.

\section{Patients and methods}

\section{Population}

Inpatient hospitalizations for AP were identified using the Nationwide Inpatient Sample (NIS) from 2004 to 2014. The NIS is the largest publicly available all-payer inpatient healthcare database in the United States, yielding nationally representative estimates of inpatient outcomes. NIS provides ICD-9-CM procedural and diagnostic codes at discharge, and visit-level details including LOS, in-hospital mortality, and timing of procedures. [14]

All visits with a diagnosis of acute pancreatitis (ICD-9-CM 577.0) were included. We excluded records with a diagnosis of chronic pancreatitis (ICD-9-CM 577.1), patients younger than 18 years old, visits without a principal diagnosis, or those with invalid diagnostic or procedural ICD-9-CM codes. We also excluded those with a diagnosis of acute cholangitis (ICD-9CM 576.2) and any records of patients who were transferred in from another institution. We selected patients with gallstone- related pancreatitis according to previously published ICD-9CM schemas. Briefly, we used any ICD-9-CM codes associated with gallstone disease [15]. Based on the revised Atlanta criteria, we defined severe acute pancreatitis (SAP) based on evidence of end-organ failure by including at least one of a number of ICD-9-CM codes associated with acute renal failure, acute respiratory failure, sepsis, and shock (Supplemental Table 1) [16-19]. We selected a population of AGPNC patients with invasive management by defining those who had ERCP, endoscopic ultrasound (EUS), and percutaneous drainage (IR) procedures, or surgical debridement of pancreas procedures on admission. (Supplemental Table 1) [20-23]. Conservative (noninvasive) management was defined as when the discharge did not contain any ERCP, EUS, IR, or surgical procedures. This study was evaluated by the Weill Cornell Medical College institutional review board and was deemed exempt (IRB\# 1706018295).

\section{Outcomes}

Our primary objective was to determine the association between in-hospital mortality and ERCP among hospitalizations for AGPNC. Our secondary objectives were to determine the association between early ERCP (on admission or 1 day after) and visit-related outcomes, including in-hospital mortality and LOS.

\section{Statistical analysis}

All data analysis was done using Stata v. 14.2 (Stata Corp., College Station, Texas, United States). Baseline characteristics were reported as mean (standard deviation) or median (interquartile range) for parametric and non-parametric variables respectively. Pearson's chi-square test or the student's $t$-test were used to evaluate differences as appropriate. Statistical significance was set at a two-tailed $P<0.05$. Data are presented as odds ratios (OR) with $95 \%$ confidence intervals in parentheses: OR (95\% confidence interval). Our analysis accounted for weighing, stratification, clustering, and change in data reporting over time.

A multivariable logistic regression model was created to determine the association between ERCP and in-hospital mortality using a backward step-wise method to eliminate variables that contributed the least to the model. We evaluated Elixhauser comorbidity index, age, sex, and presence of severe acute pancreatitis (SAP) in the multivariable logistic model, to adjust for factors that influence severity of pancreatitis [19, 24, 25]. The Elixhauser comorbidity index is a method of categorizing comorbidities of patients based on ICD-9-CM discharge codes. It can be used to predict in-house mortality [26]. Our final model included Elixhauser comorbidity index, age, and SAP.

The bootstrap method was used to perform internal validation using receiver operator curves from the native and bootstrapped model that was ran 100 times [27]. Goodness-of-fit was assessed by evaluating overdispersion (Supplemental Fig. 1) [28].

To investigate the relationship between ERCP and LOS, we created a multivariate linear regression model with natural logarithm-adjusted LOS as a dependent variable, and age, sex, presence of SAP, and presence of ERCP as independent vari- 
ables. Because of the skewed distribution of LOS, to analyze it as a continuous variable, we performed a logarithmic transformation to satisfy the assumption of normality. Given that the outcome was a log-transformed variable, we were able to report results of linear regression as a (regression coefficient) *100 to show percentage of change. Otherwise, results were reported as regression coefficient ( $95 \%$ confidence interval).

\section{Results}

There were 872,348 inpatient visits that included a diagnosis of AP. To derive the study population, we included those with gallstone-related ICD-9-CM codes and excluded any records that had codes for chronic pancreatitis $(92,821)$, acute cholangitis $(15,622)$, age younger than $18(2,177)$, those with invalid diagnostic or procedural codes (893), missing principal diagnosis $(3,060)$, and any records of patients who were transferred in from elsewhere for definitive treatment $(34,279)$, with some records containing more than a single code above. After exclusions, there were 491,011 records of patients with AGPNC eligi-

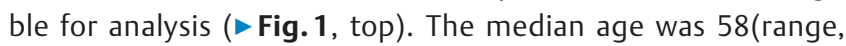
41-74) and $63 \%$ were female. This population's median Elixhauser co-morbidity index was 2 (range, 1-3). Of the patients, $30.6 \%(150,101)$ had AGPNC. Of those, $43,810(29.2 \%)$ had ERCP ( Fig. 1, bottom). Incidence of end-organ damage in the sample of patients with gallstone-related AP totaled $15.2 \%$ of discharges: acute renal failure $(8.73 \%)$, respiratory failure $(7.30 \%)$, sepsis $(5.63 \%)$, and shock $(1.56 \%)$ ( Table 1$)$. The average LOS was $5.88( \pm 6.38)$ days with median LOS of 4 days (range, 3-7).

\section{In-hospital mortality}

From 2004 to 2014, average inpatient mortality for AGPNC was 1.32 per 100 inpatient discharges. Death occurred in $0.81 \%$ of AGPNC patients with ERCP vs. $1.56 \%$ of patients with AGPNC who did not have ERCP at any time during hospitalization $(P<$ $0.0001)$. This represents a significant mortality benefit with an unadjusted OR of $0.56(0.480 .63)$. When adjusted for age, Elixhauser comorbidity index, and SAP, patients with ERCP on admission were $43 \%$ less likely to die: 0.57 (0.50 0.64) ( $>$ Table 2). Those who underwent early ERCP (within 24 hours of admission) had a mortality benefit in a univariate analysis; OR 0.69 (0.54 0.86), but not when adjusting for age, severe AP, and Elixhauser index OR 1.00 (0.79 1.28) ( Table 3). ERCP performed between hospital admission Days 3 to 9 was associated with a mortality benefit when adjusted for age, Elixhauser comorbidity index, and SAP ( $\mathbf{F i g} .2$ ).

\section{Length of stay}

Patients with AGPNC who had ERCP on admission had an average LOS of 6.73 days (6.47) while those with AGPNC who did not have ERCP had an average LOS of 5.53 days (6.30) $(P<$ $0.0001)$. ERCP performed during the hospital stay was associated with a $29 \%$ longer LOS when adjusted for age, Elixhauser comorbidity index and SAP (0.29; range, 0.28-0.30) (> Table 4). However, early ERCP (on day of admission and day after) resulted in an average LOS of 5.10 days (5.04) in the hospital vs 7.73

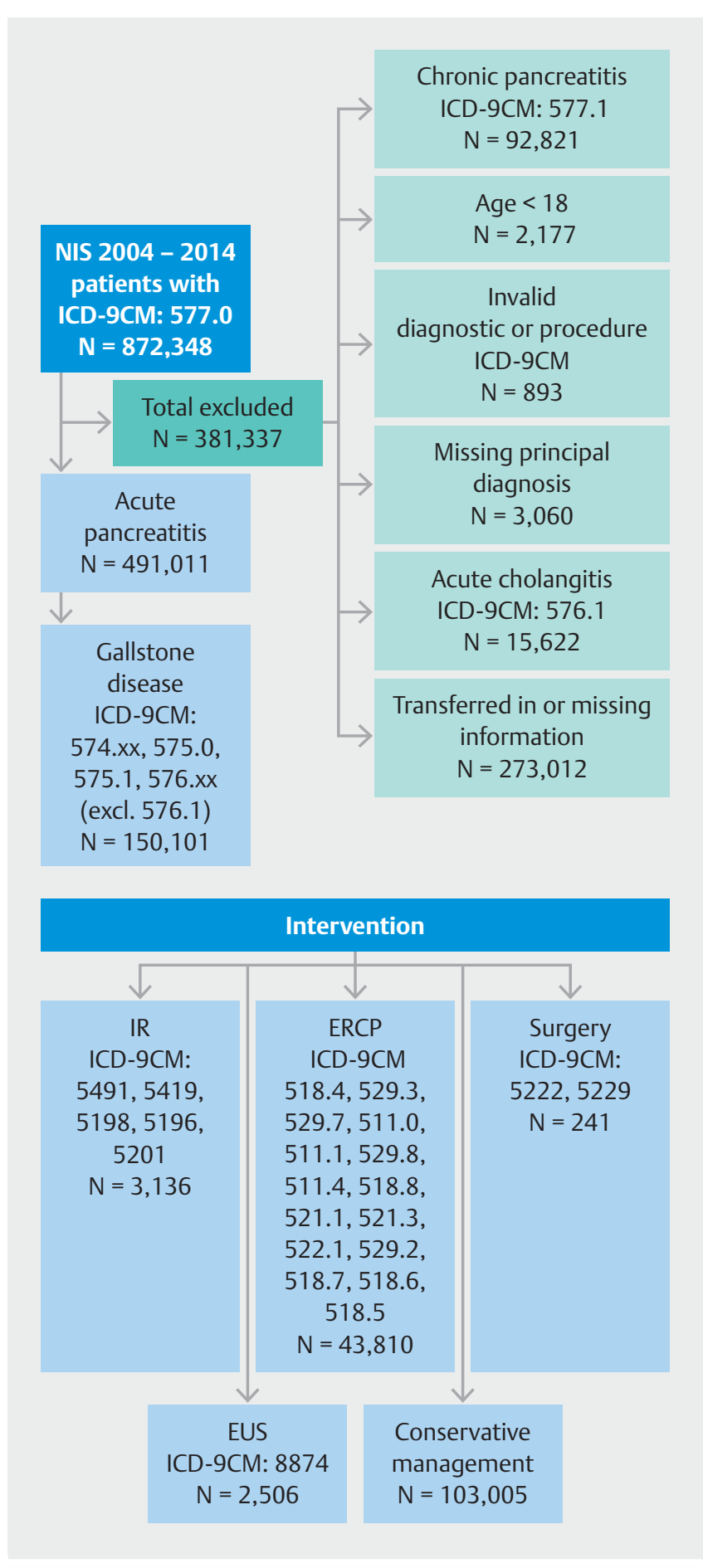

Fig. 1 The top of the figure shows population selection flowchart including exclusion criteria and excluded cases. The bottom of the figure shows management of the study population defined by ICD$9 \mathrm{CM}$ codes associated with hospitalization.

days (7.03) and corresponded to an adjusted $39 \%$ reduction in the LOS: 0.39 (range, -0.40 to -0.38 ) when compared to ERCP performed at any other time during the hospitalization $(\triangleright \mathrm{Ta}$ ble 5). 
- Table1 Baseline characteristics.

\begin{tabular}{|c|c|c|c|}
\hline Variable N (\%) & $\begin{array}{l}\text { AGPNC, overall } \\
N=150,101\end{array}$ & $\begin{array}{l}\text { AGPNC without ERCP } \\
N=106,291\end{array}$ & $\begin{array}{l}\text { AGPNC with ERCP } \\
N=43,810\end{array}$ \\
\hline Age, years mean (SD) & $56.4(19.8)$ & $56.3(19.7)$ & $56.9(20.1)$ \\
\hline Female gender & $91,751(61.1 \%)$ & $63,669(60.0 \%)$ & $28,082(64.1 \%)$ \\
\hline Elixhauser Index, median [IQR] & $2[1,4]$ & $2[1,4]$ & $2[1,3]$ \\
\hline Severe acute pancreatitis (SAP), total & $22,788(15.2 \%)$ & $16,722(15.7 \%)$ & $6,066(13.9 \%)$ \\
\hline Acute renal failure & $15,098(10.1 \%)$ & $11,423(10.8 \%)$ & $3,675(8.39 \%)$ \\
\hline Sepsis & $8,659(5.77 \%)$ & $6,126(5.76 \%)$ & $2,533(5.78 \%)$ \\
\hline Respiratory failure & $7,212(4.80 \%)$ & $5,483(5.16 \%)$ & $1,729(3.95 \%)$ \\
\hline Shock & $2,545(1.55 \%)$ & $2,026(1.91 \%)$ & $519(1.19 \%)$ \\
\hline In-hospital mortality & $2,009(1.34 \%)$ & $1,656(1.56 \%)$ & $353(0.81 \%)$ \\
\hline Length of stay, days mean (SD) & $5.89(6.38)$ & $5.54(6.31)$ & $6.73(6.41)$ \\
\hline
\end{tabular}

- Table 2 Procedural effect on inpatient mortality in gallstone pancreatitis.

\begin{tabular}{|l|l|l|l|l|}
\hline & & & Odds of death if ERCP was done any time on admission \\
\hline & Unadjusted OR (95\% Cl) & P value & Adjusted OR $\mathbf{( 9 5 \% ~ C I ) ~}$ & $\mathbf{P}$ value \\
\hline Conservative Management & Ref & NA & Ref \\
\hline ERCP & $0.56(0.480 .63)$ & $<0.001$ & $0.57(0.500 .64)$ \\
\hline Age & $1.05(1.051 .05)$ & $<0.001$ & $1.03(1.031 .04)$ \\
\hline Severe Pancreatitis & $49.4(42.956 .9)$ & $<0.001$ & $32.1(27.337 .8)$ \\
\hline Elixhauser Index & $1.47(1.441 .49)$ & $<0.001$ & $1.07(1.041 .10)$ \\
\hline ERCP, endoscopic retrograde cholangiopancreatography; Cl, confidence interval. & \\
\hline
\end{tabular}

- Table 3 Effect of early ERCP on inpatient mortality in gallstone pancreatitis.

\begin{tabular}{|l|l|l|l|l|}
\hline & & & Odds of Death if ERCP was done early \\
\hline & Univariate OR (95\%) & P value & Multivariate OR (95\%Cl) & P value \\
\hline ERCP $>$ Day 1 & Ref & NA & Ref & NA \\
\hline ERCP $\leq$ Day 0-1 & $0.69(0.540 .86)$ & $<0.001$ & $1.00(0.791 .28)$ \\
\hline Age & $1.05(1.051 .06)$ & $<0.001$ & $1.03(1.021 .04)$ \\
\hline Severe Pancreatitis & $49.4(42.956 .9)$ & $<0.001$ & $25.5(18.036 .1)$ \\
\hline Elixhauser Index & $1.47(1.441 .49)$ & $<0.001$ & $1.09(1.021 .17)$ \\
\hline ERCP, endoscopic retrograde cholangiopancreatography. & & & $<0.001$ \\
\hline
\end{tabular}

\section{Discussion}

In this study, we evaluated outcomes of AGPNC in the United States. Our results demonstrated that ERCP performed in patients with AGPNC had a mortality benefit when adjusted for age, Elixhauser comorbidity index, and SAP. However, there was no difference in survival with early ERCP in these patients.
Given lack of mortality benefit from early ERCP but an apparent benefit from ERCP overall, we hypothesized that there is an alternative time point at which endoscopic intervention can become useful in these patients. When extending the interval from admission to when ERCP was performed, the mortality benefit appeared most pronounced between hospital Days 3 and 9. Performance of ERCP 72 hours after admission has been 


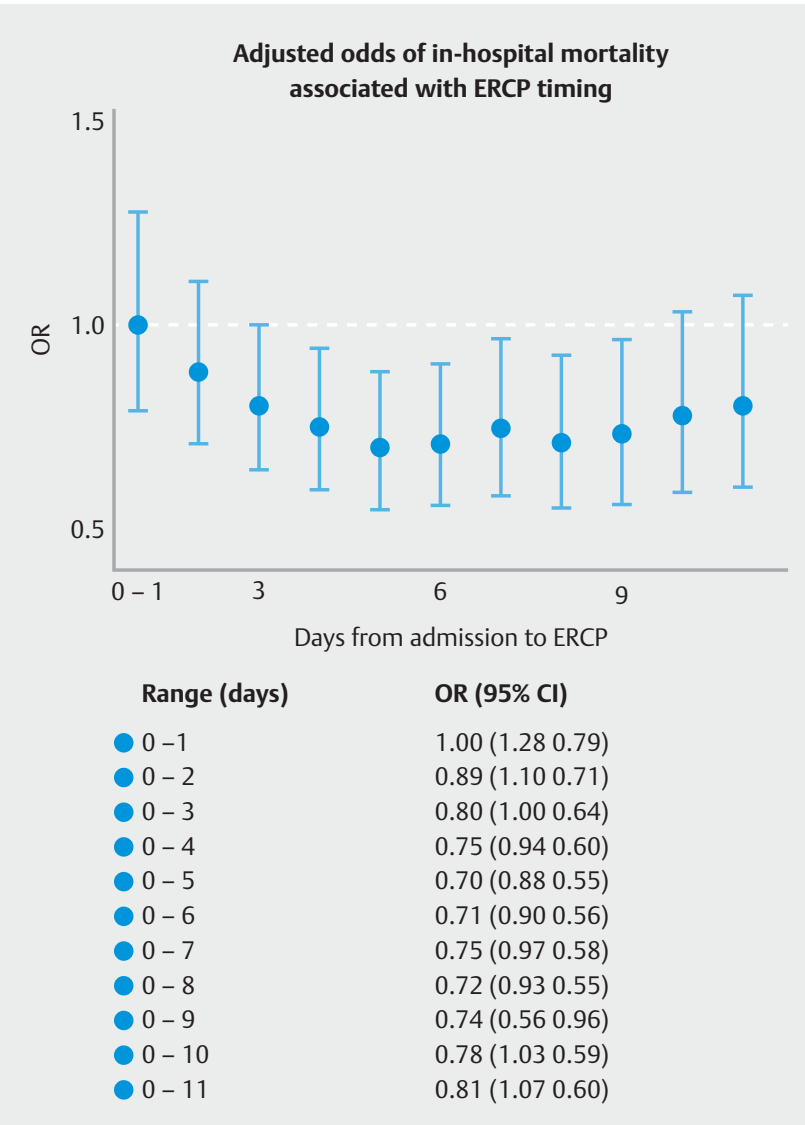

Fig. 2 This graph shows optimal timing of ERCP for reduction in in-hospital mortality, based on a comparison of patients who had early ERCP and those who had ERCP later in the course of hospitalization.

investigated before in two prospective studies, and in both, it decreased the severity of pancreatitis $[6,29]$. The other two trials that investigated use of ERCP within 72 hours of onset of symptoms have not shown any improvements over conservative management $[30,31]$. This points to the importance of resuscitation and initial acute care before the procedure, and may explain why ERCP mortality benefit becomes significant only on Day 3 of hospitalization. It is interesting that our data show no mortality benefit after Day 9 of hospitalization even after adjustment for age, sickness, and severity of pancreatitis - factors that can delay a procedure. This may mean that after a certain point, ERCP is not beneficial in regard to prevention of pancreatitis complications.

LOS can be viewed as an indirect measure of the adverse events of pancreatitis. In this study, patients with AGPNC who had ERCP had a $29 \%$ longer LOS compared to those with conservative management. Among those who had ERCP, a shorter wait time for ERCP was associated with a shorter LOS. Our data suggest that there may be an optimal time to perform ERCP for gallstone pancreatitis (to balance the decrease the LOS while improving mortality). Delayed ERCP - longer than 9 days after hospitalization - was associated with both increased mortality and increased LOS in our study. It is important to note that while there is an association between LOS and ERCP, that does not imply that ERCP causes longer hospital stays. It may just be pointing to the severity of patient illness.

Selection bias is inherent in a retrospective study design. We accounted for weighing, stratification, clustering, and change in data reporting over time. Furthermore, analysis was adjusted for age, general level of comorbidity, and inpatient level of pancreatitis severity to make conservative management and ERCP groups comparable. Longer hospital stays in patients with AGPNC who have ERCP point to the fact that this group is just as sick, if not sicker, compared to those with AGPNC who have conservative management. This strengthens our findings regarding ERCP mortality benefit.

The study had limitations. It was an analysis of the administrative claims database, and was based on ICD-9-CM discharge codes. Because ICD-9-CM diagnostic codes lack time stamps, we could not distinguish between pancreatitis complications and ERCP complications themselves. Moreover, we could not evaluate the clinical reasoning for ERCP in individual cases. No adjustment of comorbidities will remove procedural bias inherent in patients who underwent ERCP. In addition, claims data do not provide clinical information such as results of imaging, vital signs, and laboratory values, or subjective data such as pain and discomfort. Therefore, we could not classify SAP, based on BISAP or APACHE II classifications. The combination of codes used to derive in-hospital complications (sepsis, AKI, respiratory failure) did not predict mortality perfectly, as was shown in prior studies $[17,19]$; however, in our model together, $r$ they had a good predictive power. APEC (Early biliary decompression versus conservative treatment in acute biliary pancreatitis) is a well-powered multicenter Dutch randomized controlled trial for which recruitment has been completed that it is hoped willy be able to answer questions regarding optimal timing and usefulness of ERCP in a prospective fashion [32].

Management of gallstone pancreatitis involves removal of the gallbladder. In some cases, only cholecystectomy is done if there is no indication for ERCP. It would be intriguing to compare outcomes in these groups. However, performance of cholecystectomy on the same admission for gallstone pancreatitis has evolved in the past two decades. Because cholecystectomy during hospitalization for gallstone pancreatitis was not always a standard of care between 2004 and 2014, we chose not to include cholecystectomy as a variable in our analysis. It is true that inpatient cholecystectomy is an important confounding factor and inability to account for an effect from a possible cholecystectomy further limits our predictive modeling of any possible mortality benefit from ERCP. A cross-sectional study of a recent large inpatient dataset could help evaluate the relationship between inpatient cholecystectomy and gallstone pancreatitis mortality.

Interestingly, relatively few patients underwent EUS for management of gallstone pancreatitis. While the role for diagnostic and interventional EUS is constantly expanding, EUS was still a rare procedure between 2004 and 2014. As such, our dataset lacked the numbers to conduct any robust analysis of the data. 
Table 4 Procedural effect on LOS in gallstone-related pancreatitis.

\begin{tabular}{|c|c|c|c|c|c|c|}
\hline & & & & $\begin{array}{l}\text { \% Change in LOS when ERCP was } \\
\text { done at any time on the admission }\end{array}$ & & \\
\hline & Univariate OR (95\%) & S.E. & $P$ value & Multivariate OR $(95 \% \mathrm{Cl})$ & S.E. & $P$ value \\
\hline Conservative Management & Ref & & NA & Ref & & NA \\
\hline ERCP & $0.27(0.270 .28)$ & .004 & $<0.001$ & $0.29(0.280 .30)$ & .004 & $<0.001$ \\
\hline Age & $0.01(0.010 .01)$ & .000 & $<0.001$ & $0.00(0.000 .00)$ & .000 & 0.004 \\
\hline Severe pancreatitis & $0.69(0.680 .70)$ & .006 & $<0.001$ & $0.55(0.540 .56)$ & .006 & $<0.001$ \\
\hline Elixhauser Index & $0.10(0.100 .11)$ & .000 & $<0.001$ & $0.07(0.070 .07)$ & .005 & $<0.001$ \\
\hline
\end{tabular}

- Table 5 Effect of early ERCP on LOS in gallstone-related pancreatitis.

\begin{tabular}{|c|c|c|c|c|c|c|}
\hline & & & & \% change in LOS when ERCP was done early & & \\
\hline & $\begin{array}{l}\text { Univariate regression } \\
\text { coefficient (95\%) }\end{array}$ & S.E. & $P$ value & Multivariate regression coefficient $(95 \% \mathrm{Cl})$ & S.E. & $P$ value \\
\hline ERCP $>$ Day 1 & Ref & & NA & Ref & & NA \\
\hline $\mathrm{ERCP} \leq$ Day $0-1$ & $-0.44(-0.46-0.43)$ & .006 & $<0.001$ & $-0.39(-0.40-0.38)$ & .006 & $<0.001$ \\
\hline Age & $0.01(0.010 .01)$ & .000 & $<0.001$ & $0.00(0.000 .00)$ & .000 & 0.004 \\
\hline Severe pancreatitis & $0.69(0.680 .70)$ & .006 & $<0.001$ & $0.52(0.510 .55)$ & .010 & $<0.001$ \\
\hline Elixhauser Index & $0.10(0.100 .11)$ & .000 & $<0.001$ & $0.06(0.060 .06)$ & .002 & $<0.001$ \\
\hline
\end{tabular}

ERCP, endoscopic retrograde cholangiopancreatography; LOS, length of stay; S.E., standard error.

\section{Conclusion}

Treatment of patients with an acute gallstone-related pancreatitis without cholangitis is challenging. This analysis of a large administrative database suggests that ERCP during the hospital stay may be of use and that the procedure should be done early - but not too early - during a hospital stay to allow proper care and resuscitation. Further work should focus on better defining clinical subgroups of patients who may benefit from endoscopic interventions.

\section{Competing interests}

The authors declare that they have no conflict of interest.

\section{References}

[1] Peery AF, Crockett SD, Barritt AS et al. Burden of gastrointestinal, liver, and pancreatic diseases in the United States. Gastroenterology 2015; 149: 1731-1741e3

[2] Russo MW, Wei JT, Thiny MT et al. Digestive and liver diseases statistics, 2004. Gastroenterology 2004; 126: 1448-1453

[3] Hirano T, Manabe T. A possible mechanism for gallstone pancreatitis: repeated short-term pancreaticobiliary duct obstruction with exocrine stimulation in rats. Proc Soc Exp Biol Med 1993; 202: 246-252
[4] Acosta JM, Rossi R, Galli OM et al. Early surgery for acute gallstone pancreatitis: evaluation of a systematic approach. Surgery 1978; 83 : 367-370

[5] Senninger N, Moody FG, Coelho JC et al. The role of biliary obstruction in the pathogenesis of acute pancreatitis in the opossum. Surgery 1986; 99: 688-693

[6] Neoptolemos JP, Carr-Locke DL, London NJ et al. Controlled trial of urgent endoscopic retrograde cholangiopancreatography and endoscopic sphincterotomy versus conservative treatment for acute pancreatitis due to gallstones. Lancet 1988; 2: 979-983

[7] Fan ST, Lai EC, Mok FP et al. Early treatment of acute biliary pancreatitis by endoscopic papillotomy. N Engl J Med 1993; 328: 228-232

[8] Nowak A, Nowakowska-Dulawa E, Marek TA. Department of Gastroenterology, Silesian University School of Medical Sciences. et al. Final results of the prospective, randomized, controlled study on endoscopic sphincterotomy versus conventional management in acute biliary pancreatitis. Gastroenterology 1995; 108: A380

[9] Sharma VK, Howden CW. Metaanalysis of randomized controlled trials of endoscopic retrograde cholangiography and endoscopic sphincterotomy for the treatment of acute biliary pancreatitis. Am J Gastroenterol 1999; 94: 3211-3214

[10] Tse F, Yuan Y. Early routine endoscopic retrograde cholangiopancreatography strategy versus early conservative management strategy in acute gallstone pancreatitis. Cochrane Database Syst Rev; 2012: CD009779

[11] Tenner S, Baillie J, DeWitt J et al. American College of Gastroenterology Guideline: Management of Acute Pancreatitis. Am J Gastroenterol 2013; 108: 1400-1415 
[12] Working Group IAP/APA Acute Pancreatitis Guidelines. IAP/APA evidence-based guidelines for the management of acute pancreatitis. Pancreatology 2013; 13: e1-15

[13] Crockett SD, Wani S, Gardner TB et al. American Gastroenterological Association Institute Guideline on Initial Management of Acute Pancreatitis. Gastroenterology 2018; 154: 1096-1101

[14] HCUP National Inpatient Sample (NIS). Healthcare Cost and Utilization Project (HCUP). 2004-2014. Agency for Healthcare Research and Quality; Rockville, MD: http://www.hcup-us.ahrq.gov/nisoverview. jsp. Please see in: https://www.hcup-us.ahrq.gov/tech_assist/citations.jsp

[15] Chen Y, Zak Y, Hernandez-Boussard T et al. The epidemiology of idiopathic acute pancreatitis, analysis of the nationwide inpatient sample from 1998 to 2007. Pancreas 2013; 42: 1-5

[16] Banks PA, Bollen TL, Dervenis C et al. Classification of acute pancreatitis-2012: revision of the Atlanta classification and definitions by international consensus. Gut 2013; 62: 102-111

[17] Kerlin MP, Weissman GE, Wonneberger KA et al. Validation of administrative definitions of invasive mechanical ventilation across 30 intensive care units. Am J Respir Crit Care Med 2016; 194: 1548-1552

[18] Yamana H, Horiguchi H, Fushimi K et al. Comparison of procedurebased and diagnosis-based identifications of severe sepsis and disseminated intravascular coagulation in administrative data. J Epidemiol 2016; 26: 530-537

[19] Brown JR, Rezaee ME, Nichols EL et al. Incidence and in-hospital mortality of acute kidney injury (AKI) and dialysis-requiring AKI (AKI-D) after cardiac catheterization in the National inpatient sample. J Am Heart Assoc 2016; 5: e002739

[20] McNabb-Baltar J, Trinh QD, Barkun AN. Biliary drainage method and temporal trends in patients admitted with cholangitis: A national audit. Can J Gastroenterol 2013; 27: 513-518

[21] Mazen Jamal M, Yoon EJ, Saadi A et al. Trends in the utilization of endoscopic retrograde cholangiopancreatography (ERCP) in the United States. Am J Gastroenterol 2007; 102: 966-975
[22] Centers for Disease Control and Prevention, National Center for Health Statistics. ICD - ICD-9-CM - International Classification of Diseases, Ninth Revision, Clinical Modification. Classif Dis Funct Disabil 2013; 2008: 1-2

[23] Wormer BA, Swan RZ, Williams KB et al. Outcomes of pancreatic debridement in acute pancreatitis: Analysis of the nationwide inpatient sample from 1998 to 2010. Am J Surg 2014; 208: 350-362

[24] Forsmark CE, Swaroop Vege S, Wilcox CM. Acute Pancreatitis. N Engl J Med 2016; 375: 1972-1981

[25] Chen H-J, Wang J-J, Tsay W-I et al. Epidemiology and outcome of acute pancreatitis in end-stage renal disease dialysis patients: a 10-year national cohort study. Nephrol Dial Transplant 2017; 32: 1731-1736

[26] Elixhauser A, Steiner C, Harris DR et al. Comorbidity measures for use with administrative data. Med Care 1998; 36: 8-27

[27] Cleves MA. From the help desk: Comparing areas under receiver operating characteristic curves from two or more probit or logit models. Stata | 2002; 2: 301-313

[28] Suárez EL, Pérez CM, Nogueras GM et al. Biostatistics in Public Health Using STATA. CRC Press; 2016

[29] Chen P, Hu B, Wang C et al. Pilot study of urgent endoscopic intervention without fluoroscopy on patients with severe acute biliary pancreatitis in the intensive care unit. Pancreas 2010; 39: 398-402

[30] Oría A, Cimmino D, Ocampo C et al. Early endoscopic intervention versus early conservative management in patients with acute gallstone pancreatitis and biliopancreatic obstruction: A randomized clinical trial. Ann Surg 2007; 245: 10-17

[31] Fölsch UR, Nitsche R, Lüdtke R et al. Early ERCP and papillotomy compared with conservative treatment for acute biliary pancreatitis. The German Study Group on Acute Biliary Pancreatitis. N Engl J Med 1997; 336: 237-242

[32] Schepers N], Bakker OJ, Besselink MGH et al. Early biliary decompression versus conservative treatment in acute biliary pancreatitis (APEC trial): study protocol for a randomized controlled trial. Trials 2016; 17 : 5 